\title{
Cerenkov Luminescence Imaging as a Modality to Evaluate Antibody-Based PET Radiotracers
}

\author{
Jimson W. D'Souza ${ }^{1}$, Harvey Hensley ${ }^{1}$, Mohan Doss ${ }^{1,2}$, Charles Beigarten ${ }^{3}$, Michael Torgov ${ }^{3}$, Tove Olafsen ${ }^{3}$, \\ Jian Q. Yu ${ }^{1,2}$, and Matthew K. Robinson ${ }^{1}$ \\ ${ }^{1}$ Molecular Therapeutics Program, Fox Chase Cancer Center, Philadelphia, Pennsylvania; ${ }^{2}$ Nuclear Medicine, Department of \\ Diagnostic Imaging, Fox Chase Cancer Center, Philadelphia, Pennsylvania; and ImaginAb Inc., Inglewood, California
}

\begin{abstract}
Antibodies, and engineered antibody fragments, labeled with radioisotopes are being developed as radiotracers for the detection and phenotyping of diseases such as cancer. The development of antibody-based radiotracers requires extensive characterization of their in vitro and in vivo properties, including their ability to target tumors in an antigen-selective manner. In this study, we investigated the use of Cerenkov luminescence imaging (CLI) as compared with PET as a modality for evaluating the in vivo behavior of antibody-based radiotracers. Methods: The anti-prostate-specific membrane antigen (PSMA) huJ591 antibody (IgG; $150 \mathrm{kDa}$ ) and its minibody $(\mathrm{Mb} ; 80 \mathrm{kDa})$ format were functionalized with the chelator 1,4,7-triazacyclononane-1-glutaric acid-4,7-diacetic acid (NODAGA) and radiolabeled with the positron-emitting radionuclide ${ }^{64} \mathrm{Cu}$ (halflife, $12.7 \mathrm{~h}$ ). Immunoreactive preparations of the radiolabeled antibodies were injected into $\mathrm{NCr}$ nu/nu mice harboring PSMA-positive CWR22Rv1 and PSMA-negative PC-3 tumor xenografts. Tumor targeting was evaluated by both PET and CLI. Results: ${ }^{64} \mathrm{Cu}-\mathrm{NODAGA}-$ PSMA-IgG and ${ }^{64} \mathrm{Cu}-\mathrm{NODAGA}-\mathrm{PSMA}-\mathrm{Mb}$ retained the ability to bind cell surface PSMA, and both radiotracers exhibited selective uptake into PSMA-positive tumors. Under the experimental conditions used, PSMA-selective uptake of ${ }^{64} \mathrm{Cu}-$ NODAGA-PSMA-IgG and ${ }^{64} \mathrm{Cu}-$ NODAGA-PSMA-Mb was observed by CLI as early as $3 \mathrm{~h}$ after injection, with tumor-to-background ratios peaking at $24(\mathrm{lgG})$ and 16 $(\mathrm{Mb}) \mathrm{h}$ after injection. Targeting data generated by CLI correlated with that generated by PET and necropsy. Conclusion: CLI provided a rapid and simple assessment of the targeting specificity and pharmacokinetics of the antibody-based PET radiotracers that correlated well with the behavior observed by standard PET imaging. Moreover, CLI provided clear discrimination between uptake kinetics of an intact $\operatorname{lgG}$ and its small-molecular-weight derivative $\mathrm{Mb}$. These data support the use of CLI for the evaluation of radiotracer performance.
\end{abstract}

Key Words: Cerenkov luminescence imaging (CLI); ImmunoPET; antibody

J Nucl Med 2017; 58:175-180

DOI: 10.2967/jnumed.116.178780

$\mathbf{T}$ argeted cancer therapies, such as monoclonal antibodies (mAbs) and tyrosine kinase inhibitors, represent promising classes of therapeutic agents whose development is driven by our growing

Received May 26, 2016; revision accepted Jul. 11, 2016.

For correspondence or reprints contact: Matthew K. Robinson, Fox Chase Cancer Center, 333 Cottman Ave., Philadelphia, PA 19111.

E-mail: matthew.robinson@fccc.edu

Published online Aug. 18, 2016.

COPYRIGHT (C) 2017 by the Society of Nuclear Medicine and Molecular Imaging. understanding of the molecular basis of diseases. The most effective use of current and future targeted agents will require robust biomarker or imaging-based methods to stratify patients based on their predicted responses to particular therapies, making molecular imaging an indispensable tool in both drug development and clinical oncology. In contrast to anatomic imaging modalities (e.g., CT, ultrasound, or MRI) that exploit differences in the physical properties of tissue to generate image contrast, molecular imaging modalities use exogenous probes to noninvasively visualize and measure cellular processes. PET, in combination with the radiotracer ${ }^{18}$ F-FDG, monitors glucose uptake as a surrogate biomarker of malignant activity and is currently the most widely used molecular imaging probe in clinical oncology (1). Despite its broad applicability, ${ }^{18}$ F-FDG PET has demonstrated limited utility in the setting of prostate cancer (PCa) (2). Although $\mathrm{PCa}$ is a heterogeneous disease with varied clinical phenotypes, tumor tissues in up to $81 \%$ of $\mathrm{PCa}$ patients are characterized by low glucose utilization (3). In a retrospective study, ${ }^{18}$ F-FDG PET detected only $31 \%$ of local or systemic lesions in relapsed patients (4). Although it may be useful for selected populations of patients with aggressive disease (5), ${ }^{18}$ F-FDG PET was unable to effectively detect primary organconfined disease $(3,6)$ or local recurrence $(7)$ and exhibited low detection sensitivity for soft-tissue metastases (8). Sensitivity is further decreased because of bladder activity associated with radiotracer excretion and uptake in benign prostatic hyperplasia and acute prostatitis, which limit specificity. Strategies that either image tumors based on the expression of a protein biomarker (9-11) or monitor a biomarker's response to therapy (12-14) represent an attractive alternative to ${ }^{18} \mathrm{~F}$-FDG PET. This has led us, and others, to exploit the binding specificity of antibodies for use as imaging probes for the detection and characterization of prostate and other cancers.

Developing an optimized antibody-based imaging probe requires simultaneous consideration of multiple variables related to both the intrinsic properties of the probe and the cancer it is targeting. These include, but are not limited to, level and tumor enrichment of target expression, antibody affinity, antibody format, and radionuclide used for probe detection (15). Protein engineering strategies have been used on a large variety of antibodies against a diverse set of tumor antigens, to examine structure-function relationships with the goal of optimizing the pharmacokinetic and tumor-targeting properties for use as immuno-PET radiotracers (16-19). Strategies to efficiently measure the performance of different variants are critical components of any radiotracer development platform. Several positron-emitting radionuclides (e.g., ${ }^{64} \mathrm{Cu},{ }^{124} \mathrm{I},{ }^{86} \mathrm{Y},{ }^{89} \mathrm{Zr}$ ) have chemical properties and physical half-lives that are compatible with the half-lives of engineered antibody formats, which make 
them appropriate for use in immuno-PET (15). These positronemitting radionuclides decay, at least in part, through the release of $\beta$-particles with sufficient energy to result in the emission of Cerenkov radiation (20). Preclinical animal models have demonstrated that PET radiotracers elicit sufficiently high levels of Cerenkov radiation that can be detected by standard small-animal optical imaging systems $(21,22)$. This has opened up the use of Cerenkov luminescence imaging (CLI) to image PET radiotracer biodistribution. Here, we exploit a preclinical PCa model and 2 ${ }^{64} \mathrm{Cu}$-labeled immuno-PET radiotracers specific for prostate-specific membrane antigen (PSMA) to compare the utility of CLI versus PET as a means for rapid, semiquantitative estimation of radiotracer biodistribution, tumor uptake, and optimal imaging times.

\section{MATERIALS AND METHODS}

\section{Generation, Radiolabeling, and Characterization of ${ }^{64} \mathrm{Cu}-1,4,7-T r i a z a c y c l o n o n a n e-1-G l u t a r i c ~ A c i d-4,7-D i a c e t i c$ Acid (NODAGA)-PSMA-Mb and ${ }^{64} \mathrm{Cu}-$ NODAGA-PSMA-IgG}

Detailed methods describing the generation and in vitro quantification of the radiotracers are outlined in the supplemental materials (available at http://jnm.snmjournals.org).

\section{Imaging-Based Characterization of Radiotracer Performance}

A detailed description of in vivo experimental set-up, imaging parameters, and instrument settings are outlined in the supplemental materials. Mice bearing dual tumors $(n=5 /$ cohort $)$ derived from PC-3 and CWR22Rv1 cells were injected intravenously with $25 \mu \mathrm{g}$ of ${ }^{64} \mathrm{Cu}-$ NODAGA-PSMA-minibody (Mb; $5.2 \mathrm{MBq}$ ) or ${ }^{64} \mathrm{Cu}$-NODAGA-PSMAhuJ591 antibody (IgG; $3.7 \mathrm{MBq}$ ) in physiologic saline with $1 \%$ human serum albumin. Animals were anesthetized with an inhalation of a $2 \%-$ $3 \%$ isoflurane-oxygen gas mixture, and CLI data were obtained at $0.15,3$, 16,24 , and $48 \mathrm{~h}$ after injection for both antibody formats, with an additional data point at $72 \mathrm{~h}$ for ${ }^{64} \mathrm{Cu}-N O D A G A-P S M A-I g G$. Serial PET/CT images were acquired on a Siemens Biograph Truepoint PET/CT scanner at 1,24 , and $48 \mathrm{~h}$ for the ${ }^{64} \mathrm{Cu}-\mathrm{NODAGA}-\mathrm{PSMA}-\mathrm{Mb}$ and $1,24,48$, and $72 \mathrm{~h}$ for the ${ }^{64} \mathrm{Cu}-\mathrm{NODAGA}-\mathrm{PSMA}-\mathrm{IgG}$. PET images of phantoms were recorded using the same methods and instrument settings.

After acquisition of the last imaging time point, the biodistribution of radiotracers was quantified as previously described (23). Briefly, mice were euthanized by $\mathrm{CO}_{2}$ asphyxiation, and selected tissues were harvested and weighed. Radioactivity in the harvested tissues was measured in a $\gamma$-counter (Cobra Quantum; Packard Instruments), and background and decay-corrected activities in tissues were used to calculate the percentage injected dose per gram of tissue $(\% \mathrm{ID} / \mathrm{g})$.

\section{Statistical Analysis}

Statistical analysis was performed using GraphPad Prism software (version 6; GraphPad Software). Data from live cell assays, tumor uptake, and biodistribution studies were obtained and results expressed as mean \pm SEM. A $P$ value of less than 0.05 was considered statistically significant as estimated by a 2 -tailed Student $t$ test.

\section{RESULTS}

\section{In Vitro Characterization of ${ }^{64} \mathrm{Cu}-$ NODAGA-PSMA-IgG and ${ }^{64} \mathrm{Cu}-$ NODAGA-PSMA-Mb}

The purity and molecular weights of PSMA-IgG and PSMA-Mb before and after reaction with NODAGA- $N$-hydroxysuccinimide were confirmed by size-exclusion chromatography (Supplemental Fig. 1A) and sodium dodecyl sulfate-polyacrylamide gel electrophoresis under reducing and nonreducing conditions (Fig. 1A). The reaction conditions used to conjugate chelates to the anti-

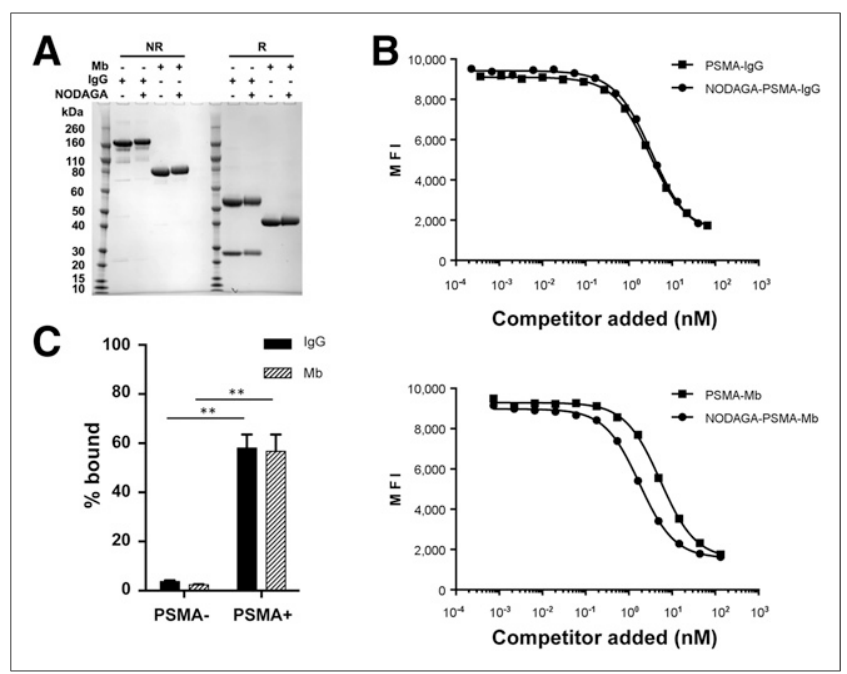

FIGURE 1. Characterization of NODAGA-PSMA-IgG and NODAGAPSMA-Mb. (A) IgG and Mb formats of anti-PSMA huJ591 antibody were analyzed by gel electrophoresis under nonreducing (NR) and reducing (R) conditions before and after conjugation of NODAGA. (B) Competition cell binding experiments demonstrate that conjugation of NODAGA did not significantly disrupt ability of either PSMA-IgG or PSMA-Mb to bind cell surface PSMA. (C) ${ }^{64} \mathrm{Cu}-\mathrm{NODAGA}-\mathrm{PSMA}-\mathrm{IgG}$ and ${ }^{64} \mathrm{Cu}-\mathrm{NODAGA}-$ PSMA-Mb bind selectively to PSMA-positive cells.

bodies yielded ratios of 2.1 and 1.8 NODAGA per protein for the $\mathrm{IgG}$ and $\mathrm{Mb}$, respectively, and were determined by reversed-phase high-performance liquid chromatography (Supplemental Fig. 1B). Conjugation of the NODAGA moieties did not result in protein aggregation as determined by size-exclusion high-performance liquid chromatography (Supplemental Fig. 1A) and had no significant impact on the ability of the antibodies to bind to PSMA expressed on the surface of cells. Overlaying titration curves demonstrate that similar concentrationdependent binding to the cell surface antigen was maintained after conjugation (Fig. 1B). Under conditions used in the competition flow experiments against AlexaFluor-488-labeled PSMA-IgG, the PSMA-IgG and NODAGA-PSMA-IgG had half maximal inhibitory concentration $\left(\mathrm{IC}_{50}\right)$ values of 3.4 and $3.0 \mathrm{nM}$, respectively. Similarly, PSMA-Mb and NODAGA-PSMA-Mb had $\mathrm{IC}_{50}$ values of 1.8 and $5.4 \mathrm{nM}$. Both protein conjugates were radiolabeled with ${ }^{64} \mathrm{CuCl}_{2}$, to greater than $98 \%$ radiochemical purity with radiochemical yields of $81 \%$ and $67 \%$ and specific activities of $146.5 \mathrm{MBq} / \mathrm{mg}(3.96 \mathrm{mCi} / \mathrm{mg})$ and $207.2 \mathrm{MBq} / \mathrm{mg}(5.6 \mathrm{mCi} / \mathrm{mg})$ for ${ }^{64} \mathrm{Cu}-\mathrm{NODAGA}-P S M A-I g G$ and ${ }^{64} \mathrm{Cu}-\mathrm{NODAGA}-\mathrm{PSMA}-\mathrm{Mb}$, respectively (Supplemental Table 1). Cell surface binding data confirmed that the radiolabeled antibodies retained sufficient antigen binding to warrant in vivo evaluation. When tested for the ability to bind cell surface PSMA in live-cell binding assays, the ${ }^{64} \mathrm{Cu}-\mathrm{NODAGA}-\mathrm{PSMA}-\mathrm{IgG}$ and ${ }^{64} \mathrm{Cu}-\mathrm{NODAGA-PSMA-Mb}$ showed an average of 15.7- and 24.2-fold increased binding, respectively, to the PSMA-positive cell line CWR22Rv1 as compared with PSMA-negative cell line PC-3 over a dose range of 20-200 ng of protein $(n=4)$. Under conditions used in these assays, the level of antigen-specific binding corresponded to immunoreactivities (average \pm SEM) of $58 \% \pm$ $10.9 \%$ for the ${ }^{64} \mathrm{Cu}-\mathrm{NODAGA}-\mathrm{PSMA}-\mathrm{IgG}$ and $56.7 \% \pm 13.6 \%$ for the ${ }^{64} \mathrm{Cu}-\mathrm{NODAGA}-\mathrm{PSMA}-\mathrm{Mb}$ (Fig. 1C; Supplemental Table 1).

\section{Comparison of CLI and PET for Imaging PSMA-Positive Tumor Xenografts}

We hypothesized that CLI would compare favorably to traditional PET imaging and provide us with a rapid, semiquantitative method 


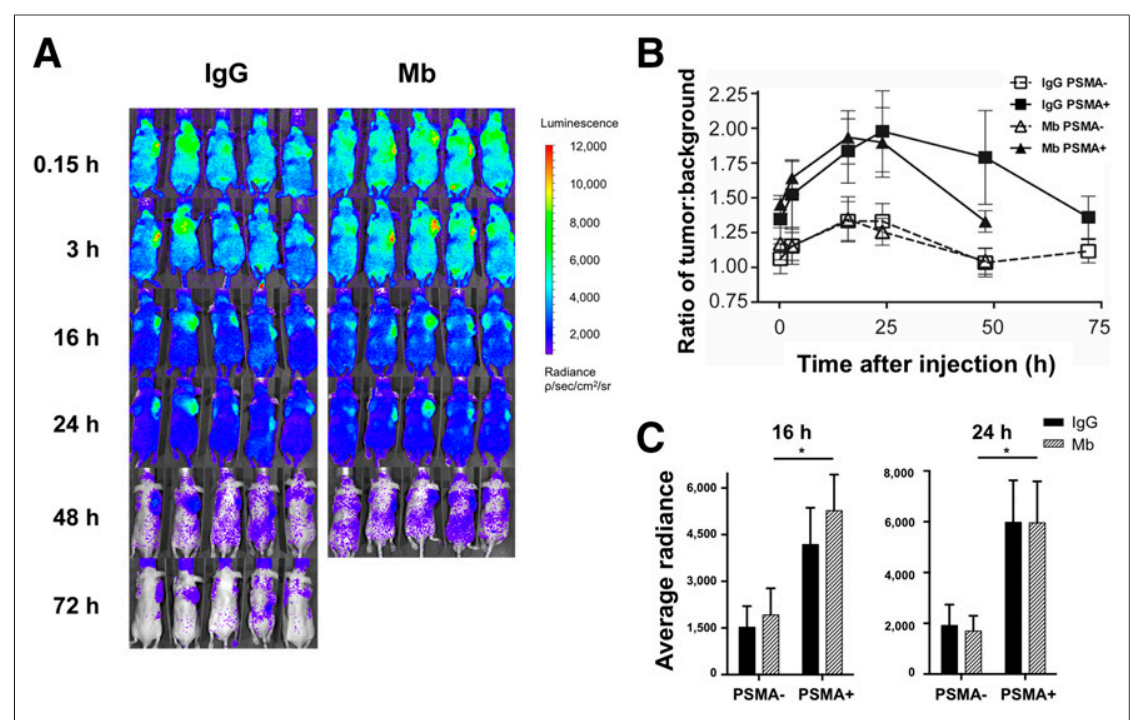

FIGURE 2. CLI with ${ }^{64} \mathrm{Cu}-\mathrm{NODAGA}-\mathrm{PSMA}-\mathrm{IgG}$ and ${ }^{64} \mathrm{Cu}-\mathrm{NODAGA}-\mathrm{PSMA}-\mathrm{Mb}$. (A) CLI-based evaluation of ${ }^{64} \mathrm{Cu}-N O D A G A-P S M A-I g G$ and ${ }^{64} \mathrm{Cu}-N O D A G A-P S M A-M b$. Luminescence was scaled to allow for direct comparison across time course. (B) Average tumor-to-background ratios across entire time course for PSMA-positive (PSMA+) and PSMA-negative (PSMA-) tumors with both radiotracers. (C) Average radiance in PSMA+ and PSMA- tumors at time of peak uptake for ${ }^{64} \mathrm{Cu}-\mathrm{NODAGA}-\mathrm{PSMA}-\mathrm{IgG}$ and ${ }^{64} \mathrm{Cu}-\mathrm{NODAGA}-\mathrm{PSMA}-\mathrm{Mb}$.

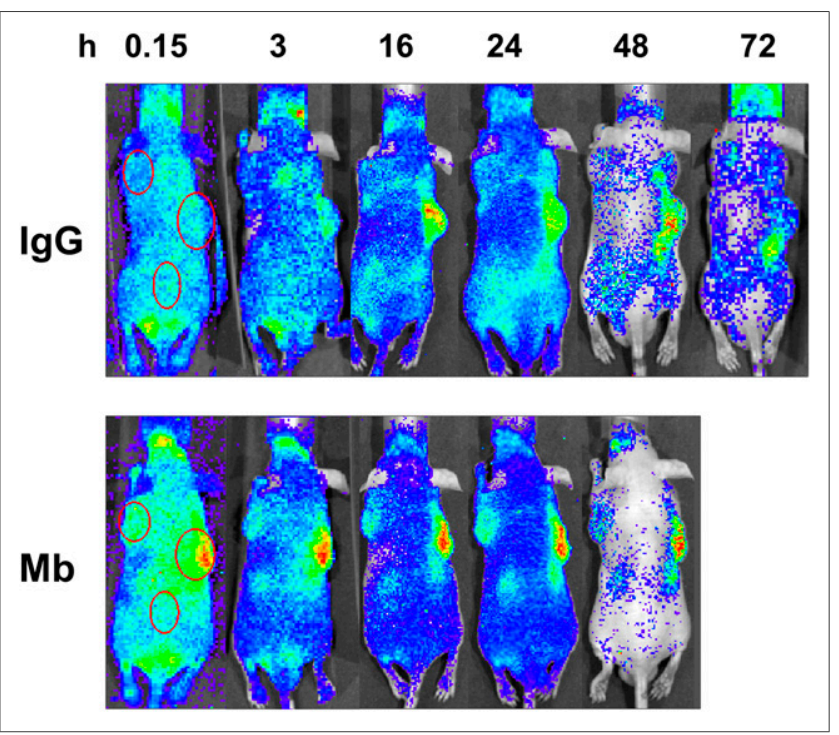

FIGURE 3. CLI. Time course analysis of representative mouse imaged with either ${ }^{64} \mathrm{Cu}-N O D A G A-P S M A-I g G$ (top) or ${ }^{64} \mathrm{Cu}-\mathrm{NODAGA-PSMA-Mb}$ (bottom). Scales were optimized at each time point to provide for maximum contrast between 22Rv1 PSMA-positive (PSMA+) (right shoulder) and PC-3 PSMA-negative (PSMA-) (left shoulder) tumors. Regions of interest over tumor and background areas are shown as ellipses in first panels of each image set.

to evaluate radiotracer performance. To accomplish this comparison of methods, we used ${ }^{64} \mathrm{Cu}-\mathrm{NODAGA-PSMA-IgG}$ and ${ }^{64} \mathrm{Cu}-$ NODAGA-PSMA-Mb as radiotracers for imaging in our preclinical model system. Previous studies have shown that the optimal signalto-background ratios are attainable within a window of 12-48 h with formats of imaging probes similar to those in this study (ViolaVillegas et al. (37); Wilks et al. (38)). The radiotracers were administered intravenously to cohorts of 5 mice harboring bilateral
PSMA-positive CWR22Rv1 and PSMAnegative PC-3 xenografts and serially imaged up to 48 and $72 \mathrm{~h}$ for the ${ }^{64} \mathrm{Cu}-\mathrm{NODAGA}$ PSMA-Mb and ${ }^{64} \mathrm{Cu}-N O D A G A-P S M A-$ $\mathrm{IgG}$, respectively. For CLI, the average radiance within regions of interest corresponding to regions covering the CWR22Rv1 and PC3 tumors as well as a region of interest covering a non-tumor-bearing region on the mid-dorsal area of the mouse were used as measures of radiotracer level within the given tissue. When the signal range across the entire time course was normalized, it allowed signal intensity within the images to depict the systemic clearance of the imaging probes. As seen in Figure 2A, selective uptake of both radiotracers into PSMA-positive CWR22Rv1 tumors, as compared with the PSMA-negative PC-3 tumors and the general background, could be observed as early as $3 \mathrm{~h}$ after injection; however, under the experimental conditions used, optimal contrast was observed at a 16- to 24-h window after injection. As seen in Figure $2 \mathrm{~B},{ }^{64} \mathrm{Cu}-\mathrm{NODAGA}-\mathrm{PSMA}-\mathrm{IgG}$ reached a maximum 2-fold tumor-to-background ratio in CWR22Rv1 tumors at $24 \mathrm{~h}$ after injection as compared with a 1.3-fold tumor-to-background level in PC-3 at the same time point. At the same time point, average radiances within tumors showed that ${ }^{64} \mathrm{Cu}-\mathrm{NODAGA-PSMA-IgG}$ exhibited a 3.1-fold selective uptake into CWR22Rv1 as compared with PC-3 tumors (Fig. 2C). Selective targeting of CWR22Rv1 tumors was maintained throughout the 72 -h time course. ${ }^{64} \mathrm{Cu}$-NODAGAPSMA-Mb reached a similar 1.9-fold peak tumor-to-background ratio in the CWR22Rv1 tumors (Fig. 2B), but consistent with its anticipated faster systemic clearance, it did so by $16 \mathrm{~h}$ after injection. Uptake into antigen-negative PC-3 tumors also reached its maximum tumor-tobackground level of 1.3 at $16 \mathrm{~h}$ after injection. A direct comparison of background-normalized uptake of ${ }^{64} \mathrm{Cu}-\mathrm{NODAGA}-\mathrm{PSMA}-\mathrm{Mb}$ into CWR22Rv1 versus PC-3 tumors showed that ${ }^{64} \mathrm{Cu}-\mathrm{NODAGA-PSMA-}$ $\mathrm{Mb}$ exhibited a 2.7-fold selectivity for antigen-positive CWR22Rv1 tumors at $16 \mathrm{~h}$ after injection (Fig. 2C). Selectivity increased to 3.5fold by $24 \mathrm{~h}$ after injection because of both increased average radiance in the CWR22Rv1 tumors and clearance of the probe from PC-3 tumors. Representative mice from both cohorts are shown in Figure 3, in which images are scaled to provide optimal contrast based on overall signal at a given time point. Here, CLI can readily visualize the antigen-dependent targeting exhibited by both radiotracers across the entire time course of the study.

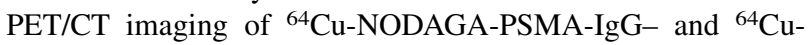
NODAGA-PSMA-Mb-injected mice corroborated the tumorselective targeting observed by CLI. Representative mice from both cohorts are depicted in Figure 4A. Quantification of radiotracer uptake via PET/CT imaging demonstrated that the decay-corrected uptake of ${ }^{64} \mathrm{Cu}-\mathrm{NODAGA}-\mathrm{PSMA}-\mathrm{IgG}$ into CWR22Rv1 tumors reached a maximum uptake of $18.7 \pm 4.7 \% \mathrm{ID}$ at $48 \mathrm{~h}$ after injection (Fig. 4B). ${ }^{64} \mathrm{Cu}-\mathrm{NODAGA-PSMA-Mb}$ reached a maximum uptake of $14.1 \pm 3.5 \% \mathrm{ID}$ in CWR22Rv1 tumors at $24 \mathrm{~h}$ after injection (Fig. 4B). Consistent with CLI results, the maximum uptake of the $\mathrm{Mb}$ occurred earlier than that observed with the IgG. In both cases, the maximum level of tumor uptake was maintained throughout the remainder of the study. Low-level uptake into PSMA-negative PC-3 


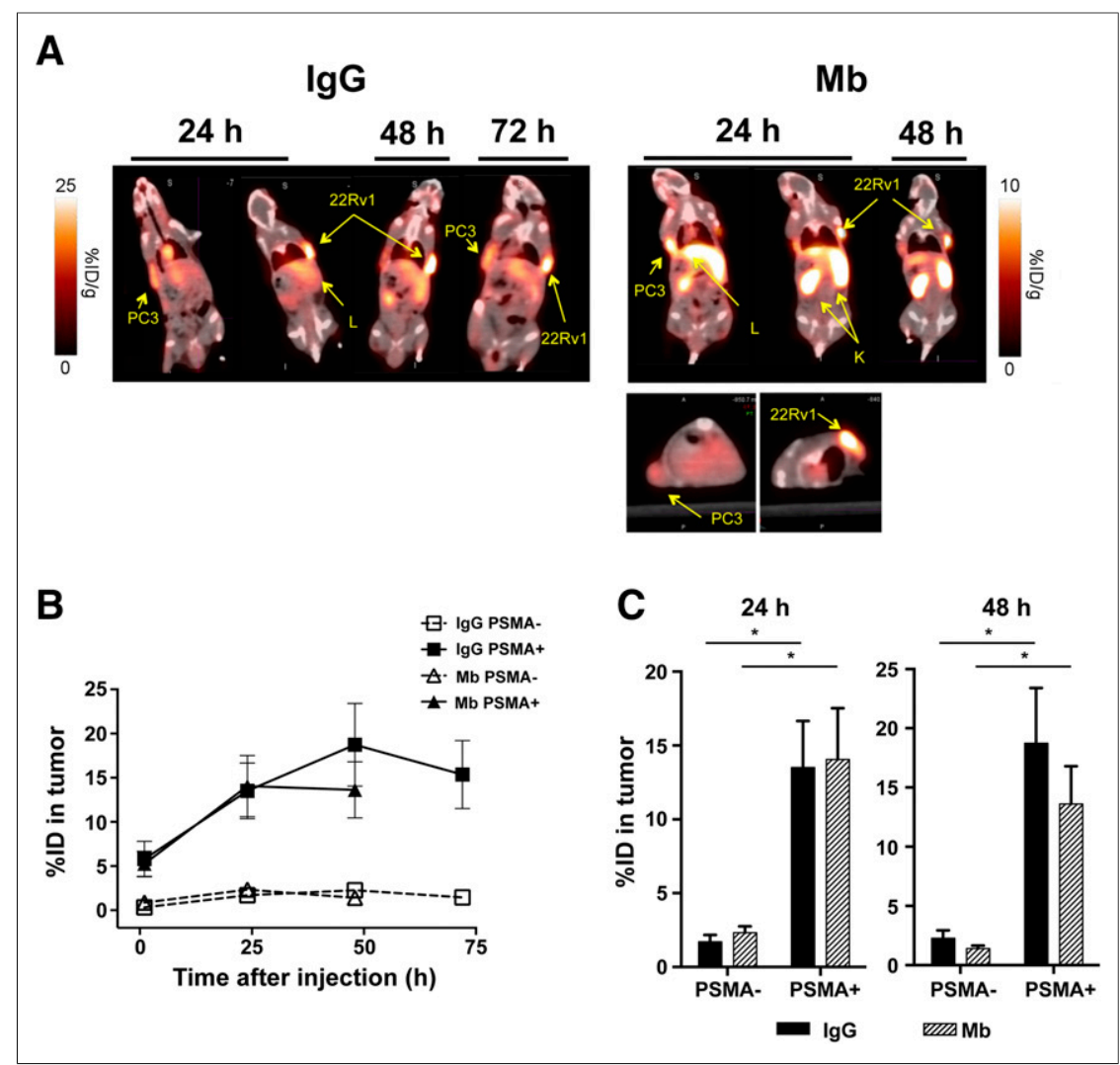

FIGURE 4. PET imaging with ${ }^{64} \mathrm{Cu}-\mathrm{NODAGA}-\mathrm{PSMA}-\mathrm{IgG}$ and ${ }^{64} \mathrm{Cu}-\mathrm{NODAGA}-\mathrm{PSMA}-\mathrm{Mb}$. (A) $\mathrm{PET} / \mathrm{CT}$ fusion images of representative mice at defined time points. Both ${ }^{64} \mathrm{Cu}-\mathrm{NODAGA}-$ PSMA-IgG and ${ }^{64} \mathrm{Cu}-\mathrm{NODAGA}-\mathrm{PSMA}-\mathrm{Mb}$ exhibited selective uptake into PSMA-positive (PSMA+) CWR22Rv1 tumor xenografts. (B) \%ID of radiotracers in PSMA+ and PSMA-negative (PSMA-) tumor xenografts over time as quantified by PET imaging. (C) PET-based uptake in PSMA + and PSMA- tumors at time of peak uptake for ${ }^{64} \mathrm{Cu}-\mathrm{NODAGA-PSMA-IgG}$ and ${ }^{64} \mathrm{Cu}-$ NODAGA-PSMA-Mb. $\mathrm{K}=$ kidneys; $\mathrm{L}=$ liver.

tumors was observed with both radiotracers over the entire time course with the exception of the 1-h time point; however, both radiotracers exhibited significantly higher $(P \leq 0.01)$ levels in the CWR22Rv1 PSMA-positive tumors even at this early time point (Fig. 4B). At time points corresponding to initial uptake peak, targeting selectivity of 8- and 6-fold for CWR22Rv1 compared with PC-3 tumors was measured by PET/CT for ${ }^{64} \mathrm{Cu}-\mathrm{NODAGA}-\mathrm{PSMA}-\mathrm{IgG}$ and ${ }^{64} \mathrm{Cu}-\mathrm{NODAGA}-\mathrm{PSMA}-\mathrm{Mb}$, respectively (Fig. 4C). The \% ID of radiotracer in PC-3 tumors for both ${ }^{64} \mathrm{Cu}-$ NODAGA-PSMA-IgG $(2.3 \pm 0.7$ vs. $1.5 \pm 0.4)$ and ${ }^{64} \mathrm{Cu}$-NODAGA-PSMA-Mb $(2.3 \pm$ 0.4 vs. $1.4 \pm 0.3)$ trended downward over the last 2 imaging time points. Although the changes in both cases failed to reach a significant level, they did result in increases in the calculated targeting selectivity. At time points corresponding to biodistribution experiments, the targeting selectivity for CWR22Rv1 versus PC-3 tumors was 10 - and 9.4-fold for ${ }^{64} \mathrm{Cu}-N O D A G A-P S M A-I g G$ and ${ }^{64} \mathrm{Cu}-$ NODAGA-PSMA-Mb, respectively.

\section{Biodistribution of Radiotracers}

Tissue distribution of the ${ }^{64} \mathrm{Cu}-N O D A G A-P S M A-I g G$ and ${ }^{64} \mathrm{Cu}-\mathrm{NODAGA}-\mathrm{PSMA}-\mathrm{Mb}$ were determined ex vivo immediately after the final imaging time points (Figs. 5A and 5B). Consistent with anticipated results, ${ }^{64} \mathrm{Cu}-\mathrm{NODAGA}-\mathrm{PSMA}-\mathrm{Mb}$ cleared from the blood more rapidly than ${ }^{64} \mathrm{Cu}-N O D A G A-P S M A-I g G$. By $24 \mathrm{~h}$ after injection, ${ }^{64} \mathrm{Cu}-\mathrm{NODAGA}-\mathrm{PSMA}-\mathrm{Mb}$ showed an activity of
$4.4 \pm 0.6 \% \mathrm{ID} / \mathrm{g}$ of blood, 10.7-fold lower than the levels observed 5 min after injection (Fig. 5B). This level was significantly lower $(P<0.008)$ than the $13.8 \pm 1.3 \%$ ID/g $(2.8-$ fold drop vs. the 5-min time point) of ${ }^{64} \mathrm{Cu}$ NODAGA-PSMA-IgG that remained in blood at the same time point (Fig. 5B). The ex vivo studies corroborated data obtained by CLI and PET that both radiotracers exhibited selective uptake into CWR22Rv1 tumor xenografts. The uptake of ${ }^{64} \mathrm{Cu}-N O D A G A-$ PSMA-IgG $(26.8 \pm 1.2 \% \mathrm{ID} / \mathrm{g})$ and ${ }^{64} \mathrm{Cu}-$ NODAGA-PSMA-Mb (21.1 $\pm 2.7 \% \mathrm{ID} / \mathrm{g})$ into the PSMA-positive tumors was statistically higher $(P<0.008)$ than the $5.99 \pm 1.6$ $\% \mathrm{ID} / \mathrm{g}$ and $4.9 \pm 1.1 \% \mathrm{ID} / \mathrm{g}$ observed in PSMA-negative tumors. This result correlated with an approximate 4.2- to 4.5-fold targeting selectivity, normalized to gram of tissue, for antigen-positive CWR22Rv1 tumors at the time of biodistribution. The radiotracers showed similar patterns of tissue distribution, with the exception of kidney uptake (Fig. 5A). Animals injected with the ${ }^{64} \mathrm{Cu}-$ NODAGA-PSMA-Mb radiotracer exhibited 2.5 -fold higher $(32 \pm 0.8$ vs. $12.9 \pm$ $1.1 \% \mathrm{ID} / \mathrm{g} ; P<0.001)$ levels of ${ }^{64} \mathrm{Cu}$ in the kidney than animals injected with ${ }^{64} \mathrm{Cu}$ NODAGA-PSMA-IgG. The increased level of signal in the kidney was observed in both CLI and PET (Figs. 2A and 4A).

\section{DISCUSSION}

Previous studies have shown that CLI can be used to noninvasively image the tumor uptake and systemic clearance of PET radiotracers (20-22). However, to our knowledge, CLI had not previously been used to evaluate, in a head-to-head manner, the performance of different antibody-based radiotracers, a strategy that has routinely been performed with PET (24-27). Therefore, the focus of these studies was to determine whether CLI can be used to distinguish between the preclinical performance of immuno-PET radiotracers that carry the same radioisotope and are targeted at the same tumor antigen but are predicted to have different clearance and tumor uptake properties. For these studies, we used full-length IgG and corresponding $\mathrm{Mb}$ formats of the huJ591 anti-PSMA antibody.

PSMA is a promising theranostic target for PCa. This type II integral plasma membrane protein undergoes constitutive internalization and is enriched on the surface of PCa cells in a manner that directly correlates with disease progression to androgen independence and metastatic spread (28-30). The clinical utility of antibody-based imaging for PCa was first established with the radiotracer Capromab Pendetide (ProstaScint), the murine mAb 7E11-C5.3 (31) conjugated to the linker-chelator glycyl-tyrosyl-( $N, \varepsilon$-diethylenetriaminepentaacetic acid) lysine hydrochloride (GYK-DTPA-HCl) and radiolabeled with ${ }^{111} \mathrm{In}$. ${ }^{111} \mathrm{In}$-Capromab demonstrated a sensitivity superior to conventional anatomic imaging techniques for detecting lymph node metastases $(32,33)$. However, the radiotracer binds to an epitope that is located on the intracellular domain of PSMA, thus its targeting specificity is limited to necrotic and apoptotic cells in the tumors. 


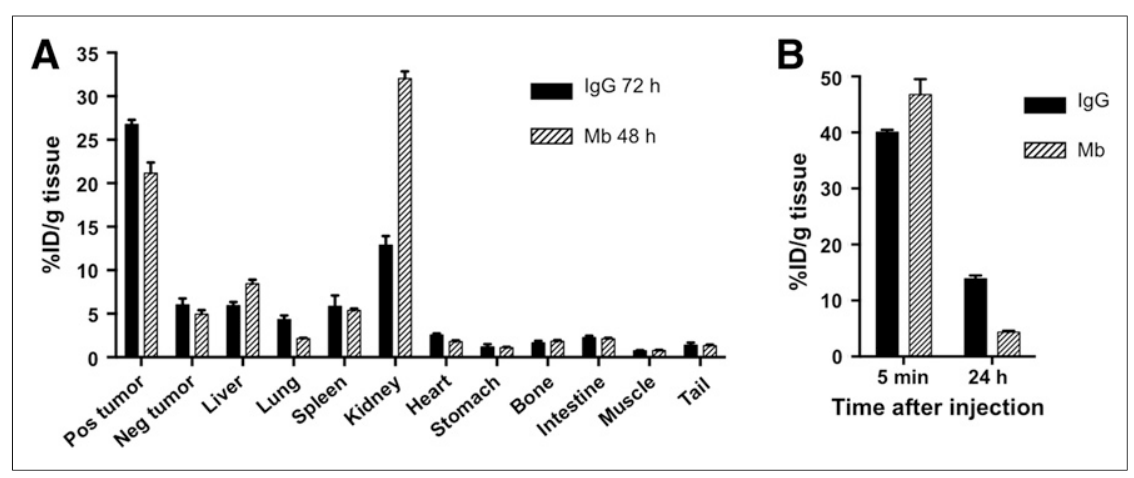

FIGURE 5. Biodistribution of ${ }^{64} \mathrm{Cu}-N O D A G A-P S M A-I g G$ and ${ }^{64} \mathrm{Cu}-N O D A G A-P S M A-M b .(A)$ Biodistribution of ${ }^{64} \mathrm{Cu}-N O D A G A-P S M A-I g G$ and ${ }^{64} \mathrm{Cu}-N O D A G A-P S M A-M b$ radiotracers at 72 and $48 \mathrm{~h}$ after injection, respectively, demonstrating statistically significant selective uptake into CWR22Rv1 PSMA-positive (PSMA+) vs. PC-3 PSMA-negative (PSMA-) tumors. (B) ${ }^{64} \mathrm{Cu}-$ NODAGA-PSMA-Mb cleared more rapidly from blood than ${ }^{64} \mathrm{Cu}-\mathrm{NODAGA}-P S M A-\lg$. ratio of ${ }^{64} \mathrm{Cu}-\mathrm{NODAGA-PSMA-IgG}$ in CWR22Rv1 versus PC-3 tumors was measured by PET, the radiotracer exhibited a 10-fold selective uptake into antigen-positive tumors at $72 \mathrm{~h}$ after injection. Similarly, ${ }^{64} \mathrm{Cu}-N O D A G A-P S M A-M b$ exhibited a 9.4-fold selective uptake into the CWR22Rv1 tumors at $48 \mathrm{~h}$ after injection. These levels of selectivity are consistent with results obtained by biodistribution if absolute levels of radiotracers (\%ID) in antigen-positive versus antigen-negative tumors are compared. If this is done, ${ }^{64} \mathrm{Cu}-$ NODAGA-PSMA-IgG exhibited a 11.4-fold and ${ }^{64} \mathrm{Cu}-\mathrm{NODAGA}-\mathrm{PSMA}-\mathrm{Mb}$ exhibited a 14-fold selectivity for PSMA-positive CWR22Rv1 tumors as compared with antigennegative PC-3 tumors. Although qualitatively

This limits the probe's overall sensitivity for detecting progressive disease and led to the isolation and characterization of novel murine mAbs specific for the extracellular domain of PSMA $(31,34)$. Subsequent humanization of the mAb J591 (huJ591) facilitated its testing as a second-generation anti-PSMA mAb for imaging and therapy (35). Expanded clinical trials are under way to evaluate the ability of ${ }^{89} \mathrm{Zr}$-DFO-huJ591 to function as a radiotracer in patients with $\mathrm{PCa}$ (ClinicalTrials.gov NCT01543659) and glioblastoma multiforme (NCT02410577). However, the pharmacokinetics and tumor-targeting properties of an imaging radiotracer are not necessarily equivalent to those of an intact IgG (15). Although immuno-PET with ${ }^{89} \mathrm{Zr}$-DFOhuJ591 can image PSMA-positive lesions in patients, the optimal imaging time was observed at $7 \mathrm{~d}$ after injection (36). Significant effort is ongoing to exploit alternative antibody-engineering strategies to generate antibody-based fragments optimized to function as improved radiotracers for detecting PSMA-positive lesions. This effort is exemplified by the work of Lewis et al. comparing the preclinical imaging properties of ${ }^{89} \mathrm{Zr}$-DFO-huJ591 IgG with those of ${ }^{89} \mathrm{Zr}$ labeled minibody and cys-diabody formats (37). Similarly, Kampmeier et al. have evaluated ${ }^{99 \mathrm{~m}}$ Tc-labeled J591 diabody as a SPECT radiotracer (39). Clinical trials are currently under way to evaluate the efficacy of the ${ }^{89} \mathrm{Zr}$-labeled minibody (ClinicalTrials.gov \#NCT01923727) formats for detecting PSMA-positive tumors.

As stated above, the ultimate goal of our study was to evaluate the potential of CLI for preclinical characterization of PET radiotracers. In general agreement with our hypothesis, CLI-based analysis of ${ }^{64} \mathrm{Cu}$-NODAGA-PSMA-IgG and ${ }^{64} \mathrm{Cu}-\mathrm{NODAGA-PSMA-Mb}$ performance in our preclinical models of $\mathrm{PCa}$ provided results that were consistent with results from PET obtained in the same animals at similar time points. The relatively short range of the Cerenkov photon transport in tissue does restrict this approach to small animals or near the surface for human use. Despite the low tissue penetration depth of some of the Cerenkov photons, CLI has desirable applications for both medical value and biologic insight (40). To evaluate the quantitative nature of both imaging modalities, we corroborated the antigen-dependent uptake measured by imaging at the final time point with data obtained by biodistribution. As exemplified in Figure 5A, radiotracer uptake in biodistribution studies is typically normalized to organ or tumor weight and presented as \% ID/g of tissue. In this study, both ${ }^{64} \mathrm{Cu}-N O D A G A-P S M A-I g G$ and ${ }^{64} \mathrm{Cu}-N O D A G A-P S M A-M b$ exhibited approximately a 4.5-fold selective targeting of CWR22Rv1 versus PC-3 tumors (Fig. 5A). However, tumor uptake measured by PET and CLI represent absolute levels of ${ }^{64} \mathrm{Cu}$ in tumors. When the similar results were obtained with CLI, decay-corrected, backgroundsubtracted average radiance of PSMA-positive tumors relative to PSMA-negative tumors showed only a 3-fold selectivity with ${ }^{64} \mathrm{Cu}$-NODAGA-PSMA-IgG and an 8-fold selectivity with ${ }^{64} \mathrm{Cu}-$ NODAGA-PSMA-Mb at the final time points.

In our studies, ${ }^{64} \mathrm{Cu}-\mathrm{NODAGA-PSMA-IgG}$ and ${ }^{64} \mathrm{Cu}-\mathrm{NODAGA-}$ PSMA-Mb performed as anticipated based on previous findings with ${ }^{89} \mathrm{Zr}$-labeled versions of the antibodies. Altering the format did not appreciably change the ability of the antibodies to bind cell surface antigen in vitro or to selectively target PSMA-positive tumors compared with PSMA-negative tumors in vivo after systemic administration. Consistent with its smaller size, ${ }^{64} \mathrm{Cu}-$ NODAGA-PSMA-Mb cleared from the blood more rapidly than ${ }^{64} \mathrm{Cu}$-NODAGA-PSMA-IgG. This increased clearance rate correlated with an overall decrease in absolute antigen-specific tumor uptake without affecting the ratio of antigen-positive to antigennegative tumor uptake. Somewhat surprising was the level of kidney uptake observed in these studies with ${ }^{64} \mathrm{Cu}$-NODAGA-PSMA$\mathrm{Mb}$. The predicted molecular weight of ${ }^{64} \mathrm{Cu}-\mathrm{NODAGA-PSMA-Mb}$ is above the threshold for renal clearance, but levels of approximately $30 \% \mathrm{ID} / \mathrm{g}$ tissue were observed in kidneys on necropsy at 48 $\mathrm{h}$ after injection. This result contrasts with 7-10\% ID/g observed in analogous studies performed with ${ }^{89} \mathrm{Zr}$-labeled huJ591-Mb (37), but are closely aligned with the results we obtained with ${ }^{64} \mathrm{Cu}-$ NODAGA-PSMA-IgG at $72 \mathrm{~h}$ after injection $(\sim 15 \% \mathrm{ID} / \mathrm{g})$. The exact reason for the increased kidney uptake exhibited by ${ }^{64} \mathrm{Cu}-$ NODAGA-PSMA-Mb in these studies is unclear but may relate to the animal model used. In similar studies, the level of kidney uptake was observed to depend on mouse strain used (ImaginAb, unpublished data). However, we cannot rule out that the increased kidney uptake was due to differences in in vivo stability of the radiotracers or to the lower radiochemical purity of the ${ }^{64} \mathrm{Cu}$ NODAGA-PSMA-Mb radiotracer than ${ }^{64} \mathrm{Cu}-N O D A G A-P S M A-I g G$ (98.8\% vs. $99.7 \%$ ). Doses were also based on mass and not adjusted for the molecular weight of the radiotracers, resulting in approximately twice as many moles of ${ }^{64} \mathrm{Cu}-\mathrm{NODAGA}-\mathrm{PSMA}-\mathrm{Mb}$ being injected compared with ${ }^{64} \mathrm{Cu}-\mathrm{NODAGA}-\mathrm{PSMA}-\mathrm{IgG}$. Both CLI and PET showed increased kidney uptake of ${ }^{64} \mathrm{Cu}-N O D A G A-P S M A-$ $\mathrm{Mb}$ as compared with ${ }^{64} \mathrm{Cu}-\mathrm{NODAGA}-\mathrm{PSMA}-\mathrm{IgG}$, because kidneys were readily visible in Cerenkov luminescence images within $16 \mathrm{~h}$ after injection of the ${ }^{64} \mathrm{Cu}$-NODAGA-PSMA-Mb (Fig. 4). This finding further supports the main goal of this study to compare CLI with PET. 


\section{CONCLUSION}

PET reconstructs the distribution of the radiotracers in 3 dimensions and provides an absolute measurement of tracer concentration, whereas CLI is based on surface radiance detected from sources at a variety of depths. Although the results from the 2 modalities are quantitatively different, the overall trends with both radiotracers are in accordance, showing that CLI can substitute for PET in providing an assessment of the targeting specificity and pharmacodynamics of antibody-based radiotracers. Considering the rapidity, simplicity, and low cost with which Cerenkov luminescence images can be obtained, our work supports the use of CLI for the evaluation of radiotracer performance.

\section{DISCLOSURE}

Work was supported by the National Institutes of Health grants CA181868 (Matthew K. Robinson) and CA06927 (FCCC CCSG). The work was also supported in part by a research grant from Imagin $\mathrm{Ab}$ to Matthew K. Robinson. Charles Beigarten, Tove Olafsen, and Michael Torgov are employees of ImaginAb and contributed to this manuscript by preparing NODAGA-PSMA-Mb and NODAGA-PSMA-IgG, analyzing data, and critically reviewing the manuscript. No other potential conflict of interest relevant to this article was reported.

\section{ACKNOWLEDGMENTS}

We acknowledge the expert technical contributions of the FCCC small-animal imaging and laboratory animal facilities. We also thank Jean Gudas, for critical review of the manuscript.

\section{REFERENCES}

1. Chen K, Chen X. Positron emission tomography imaging of cancer biology: current status and future prospects. Semin Oncol. 2011;38:70-86.

2. Lee ST, Lawrentschuk N, Scott AM. PET in prostate and bladder tumors. Semin Nucl Med. 2012;42:231-246.

3. Effert PJ, Bares R, Handt S, Wolff JM, Bull U, Jakse G. Metabolic imaging of untreated prostate cancer by positron emission tomography with 18 fluorine-labeled deoxyglucose. J Urol. 1996;155:994-998.

4. Schöder H, Herrmann K, Gonen M, et al. 2- $\left[{ }^{18} \mathrm{~F}\right]$ fluoro-2-deoxyglucose positron emission tomography for the detection of disease in patients with prostate-specific antigen relapse after radical prostatectomy. Clin Cancer Res. 2005;11:4761-4769.

5. Schöder H, Larson SM. Positron emission tomography for prostate, bladder, and renal cancer. Semin Nucl Med. 2004;34:274-292.

6. Liu IJ, Zafar MB, Lai YH, Segall GM, Terris MK. Fluorodeoxyglucose positron emission tomography studies in diagnosis and staging of clinically organ-confined prostate cancer. Urology. 2001;57:108-111.

7. Hofer C, Laubenbacher C, Block T, Breul J, Hartung R, Schwaiger M. Fluorine18-fluorodeoxyglucose positron emission tomography is useless for the detection of local recurrence after radical prostatectomy. Eur Urol. 1999;36:31-35.

8. Lawrentschuk N, Davis ID, Bolton DM, Scott AM. Positron emission tomography and molecular imaging of the prostate: an update. BJU Int. 2006;97:923-931.

9. Divgi CR, Uzzo RG, Gatsonis C, et al. Positron emission tomography/computed tomography identification of clear cell renal cell carcinoma: results from the REDECT trial. J Clin Oncol. 2013;31:187-194.

10. Scott AM, Lee FT, Tebbutt N, et al. A phase I clinical trial with monoclonal antibody ch806 targeting transitional state and mutant epidermal growth factor receptors. Proc Natl Acad Sci USA. 2007;104:4071-4076.

11. Mortimer JE, Bading JR, Colcher DM, et al. Functional imaging of human epidermal growth factor receptor 2-positive metastatic breast cancer using ${ }^{64} \mathrm{Cu}$-DOTA-trastuzumab PET. J Nucl Med. 2014;55:23-29.

12. Oude Munnink TH, Korte MA, Nagengast WB, et al. ${ }^{89} \mathrm{Zr}$-trastuzumab PET visualises HER2 downregulation by the HSP90 inhibitor NVP-AUY922 in a human tumour xenograft. Eur J Cancer. 2010;46:678-684.

13. Smith-Jones PM, Solit D, Afroze F, Rosen N, Larson SM. Early tumor response to Hsp90 therapy using HER2 PET: comparison with ${ }^{18}$ F-FDG PET. J Nucl Med. 2006;47:793-796.
14. Reddy S, Shaller CC, Doss M, et al. Evaluation of the anti-HER2 C6.5 diabody as a PET radiotracer to monitor HER2 status and predict response to trastuzumab treatment. Clin Cancer Res. 2011;17:1509-1520.

15. Reddy S, Robinson MK. Immuno-positron emission tomography in cancer models. Semin Nucl Med. 2010;40:182-189.

16. Kenanova V, Olafsen T, Crow DM, et al. Tailoring the pharmacokinetics and positron emission tomography imaging properties of anti-carcinoembryonic antigen single-chain Fv-Fc antibody fragments. Cancer Res. 2005;65:622-631.

17. Li L, Yazaki PJ, Anderson A, et al. Improved biodistribution and radioimmunoimaging with poly(ethylene glycol)-DOTA-conjugated anti-CEA diabody. $\mathrm{Bi}$ oconjug Chem. 2006;17:68-76.

18. Olafsen T, Cheung CW, Yazaki PJ, et al. Covalent disulfide-linked anti-CEA diabody allows site-specific conjugation and radiolabeling for tumor targeting applications. Protein Eng Des Sel. 2004;17:21-27.

19. Wu AM. Engineering multivalent antibody fragments for in vivo targeting. Methods Mol Biol. 2004;248:209-225.

20. Ruggiero A, Holland JP, Lewis JS, Grimm J. Cerenkov luminescence imaging of medical isotopes. J Nucl Med. 2010;51:1123-1130.

21. Boschi F, Calderan L, D'Ambrosio D, et al. In vivo ${ }^{18} \mathrm{~F}-\mathrm{FDG}$ tumour uptake measurements in small animals using Cerenkov radiation. Eur J Nucl Med Mol Imaging. 2011;38:120-127.

22. Spinelli AE, D'Ambrosio D, Calderan L, Marengo M, Sbarbati A, Boschi F. Cerenkov radiation allows in vivo optical imaging of positron emitting radiotracers. Phys Med Biol. 2010;55:483-495.

23. Robinson MK, Doss M, Shaller C, et al. Quantitative immuno-positron emission tomography imaging of HER2-positive tumor xenografts with an iodine-124 labeled anti-HER2 diabody. Cancer Res. 2005;65:1471-1478.

24. Miller J, Doss M, McQuillen R, et al. Impact of expression system on the function of the C6.5 diabody PET radiotracer. Tumour Biol. 2012;33:617-627.

25. Olafsen T, Betting D, Kenanova VE, et al. Recombinant anti-CD20 antibody fragments for small-animal PET imaging of B-cell lymphomas. J Nucl Med. 2009;50:1500-1508.

26. Stillebroer AB, Franssen GM, Mulders PF, et al. ImmunoPET imaging of renal cell carcinoma with ${ }^{124} \mathrm{I}$ - and ${ }^{89} \mathrm{Zr}$-labeled anti-CAIX monoclonal antibody cG250 in mice. Cancer Biother Radiopharm. 2013;28:510-515.

27. Cheal SM, Punzalan B, Doran MG, et al. Pairwise comparison of ${ }^{89} \mathrm{Zr}$ - and ${ }^{124} \mathrm{I}$-labeled cG250 based on positron emission tomography imaging and nonlinear immunokinetic modeling: in vivo carbonic anhydrase IX receptor binding and internalization in mouse xenografts of clear-cell renal cell carcinoma. Eur J Nucl Med Mol Imaging. 2014;41:985-994.

28. Silver DA, Pellicer I, Fair WR, Heston WD, Cordon-Cardo C. Prostate-specific membrane antigen expression in normal and malignant human tissues. Clin Cancer Res. 1997;3:81-85.

29. Mhawech-Fauceglia P, Zhang S, Terracciano L, et al. Prostate-specific membrane antigen (PSMA) protein expression in normal and neoplastic tissues and its sensitivity and specificity in prostate adenocarcinoma: an immunohistochemical study using mutiple tumour tissue microarray technique. Histopathology. 2007;50:472-483.

30. Ghosh A, Heston WD. Tumor target prostate specific membrane antigen (PSMA) and its regulation in prostate cancer. J Cell Biochem. 2004;91:528-539.

31. Chang SS, Reuter VE, Heston WD, Bander NH, Grauer LS, Gaudin PB. Five different anti-prostate-specific membrane antigen (PSMA) antibodies confirm PSMA expression in tumor-associated neovasculature. Cancer Res. 1999;59:3192-3198.

32. Polascik TJ, Manyak MJ, Haseman MK, et al. Comparison of clinical staging algorithms and 111indium-capromab pendetide immunoscintigraphy in the prediction of lymph node involvement in high risk prostate carcinoma patients. Cancer. 1999;85:1586-1592.

33. Manyak MJ, Hinkle GH, Olsen JO, et al. Immunoscintigraphy with indium-111capromab pendetide: evaluation before definitive therapy in patients with prostate cancer. Urology. 1999;54:1058-1063.

34. Liu H, Moy P, Kim S, et al. Monoclonal antibodies to the extracellular domain of prostate-specific membrane antigen also react with tumor vascular endothelium. Cancer Res. 1997;57:3629-3634.

35. Nanus DM, Milowsky MI, Kostakoglu L, et al. Clinical use of monoclonal antibody HuJ591 therapy: targeting prostate specific membrane antigen. $J$ Urol. 2003;170:S84-88; discussion S88-89.

36. Pandit-Taskar N, O'Donoghue JA, Beylergil V, et al. ${ }^{89} \mathrm{Zr}$-huJ591 immuno-PET imaging in patients with advanced metastatic prostate cancer. Eur J Nucl Med Mol Imaging. 2014;41:2093-2105.

37. Viola-Villegas NT, Sevak KK, Carlin SD, et al. Noninvasive imaging of PSMA in prostate tumors with ${ }^{89} \mathrm{Zr}$-labeled huJ591 engineered antibody fragments: the faster alternatives. Mol Pharm. 2014;11:3965-3973.

38. Wilks MQ, Knowles SM, Wu AM, et al. Improved modeling of in vivo kinetics of slowly diffusing radiotracers for tumor imaging. J Nucl Med. 2014;55:1539-1544.

39. Kampmeier F, Williams JD, Maher J, Mullen GE, Blower PJ. Design and preclinical evaluation of a ${ }^{99 \mathrm{~m}} \mathrm{Tc}$-labelled diabody of mAb J591 for SPECT imaging of prostate-specific membrane antigen (PSMA). EJNMMI Res. 2014;4:13-22.

40. Tanha K, Pashazadeh AM, Pogue BW. Review of biomedical Cerenkov luminescence imaging applications. Biomed Opt Express. 2015;6:3053-3065. 